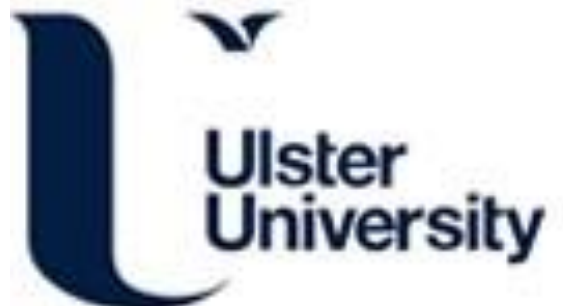

\section{Carriers Multiplication in Neighboring Surfactant-free Silicon Nanocrystals Produced by $3 \mathrm{D}$-surface Engineering in Liquid Medium}

Svrcek, V., Mariotti, D., Matsubara, K., \& Kondo, M. (2012). Carriers Multiplication in Neighboring Surfactant-free Silicon Nanocrystals Produced by 3D-surface Engineering in Liquid Medium. In Unknown Host Publication IEEE.

Link to publication record in Ulster University Research Portal

\section{Published in:}

Unknown Host Publication

Publication Status:

Published (in print/issue): 08/06/2012

\section{Document Version}

Publisher's PDF, also known as Version of record

\section{General rights}

Copyright for the publications made accessible via Ulster University's Research Portal is retained by the author(s) and / or other copyright owners and it is a condition of accessing these publications that users recognise and abide by the legal requirements associated with these rights.

\section{Take down policy}

The Research Portal is Ulster University's institutional repository that provides access to Ulster's research outputs. Every effort has been made to ensure that content in the Research Portal does not infringe any person's rights, or applicable UK laws. If you discover content in the Research Portal that you believe breaches copyright or violates any law, please contact pure-support@ulster.ac.uk. 


\title{
Carriers Multiplication in Neighboring Surfactant-free Silicon Nanocrystals Produced by 3D-surface Engineering in Liquid Medium.
}

\author{
Vladimir Svrcek ${ }^{1}$, Davide Mariotti ${ }^{3}$, Koiji Matsubara ${ }^{1}$ and Michio Kondo ${ }^{1}$ \\ ${ }^{1}$ Research Center for Photovoltaics, National Institute of Advanced Industrial Science and Technology \\ (AIST), Central 2, Umezono 1-1-1, Tsukuba, 305-8568, Japan \\ ${ }^{3}$ Nanotechnology \& Integrated Bio-Engineering Centre (NIBEC), University of Ulster, UK
}

\begin{abstract}
Carriers multiplication in silicon nanocrystals (Si-ncs) in a one promising eefect to considerably enhance conversion efficiency of solar cells that can overcome theoretical limits. A close proximity of Si-ncs is an essential factor for carrier multiplication due to the separated quantum cutting effect. In this study we present results on investigation of 3-dimensional (3D) surface engineering of Si-ncs directly in water. Thus at the same time allow close proximity Si-ncs without of using any surfactant. The approach is based on ns laser treatment of Si-ncs dispersed in liquid solution. We explore the excitation wavelength dependence of photoluminescence quantum yield (ratio of the number of emitted and absorbed photons) for $\mathrm{Si}$-ncs as prepared and surface engineered by ns laser processing. Our results suggest that close proximity of Si-ncs in spherical particles induced by laser processing might enhance also carriers multiplication.
\end{abstract}

Index Terms - silicon nanocrystals, carrier multiplication, surface engineering.

\section{INTRODUCTION}

The observation of highly efficient carrier multiplication (CM) has been observed in carbon nanotubes [1] and a range of other semiconducting nanocrystals including silicon nanocrystals (Si-ncs) [2, 3]. Si-ncs in particular, represent a promising class of environmentally friendly nanomaterials that can lead to improved solar cells efficiencies due to $\mathrm{CM}$ through closely-packed Si-ncs [3-6]. In the past decade, research in Si-ncs resulted in the study of various nanoscale properties that can be particularly useful for photovoltaic applications. Physical phenomena of major interest are multiple exciton generation (MEG) in single/isolated Si-ncs and carrier multiplication (CM) across Si-ncs in close proximity to each other. The control of surface characteristics for quantum confined structures is highly important to achieve accurate measurements of the nanocrystals properties (e.g. MEG) and to allow for successful device integration (e.g. in solar cell structures). Our investigations are focused on viable methodologies for Si-ncs surface engineering without the need of large organic molecules or surfactants. In particular two different approaches that allow 3-dimenstional (3D) surface engineering of doped Si-ncs directly in liquid medium (e.g. water) have been recently presented $[7,8]$. The first technique is based on nanosecond laser (ns-L) processing and the second is based on a DC atmospheric-pressure plasma treatment of Si- ncs dispersed in liquid media. Both techniques have produced promising results that allow for tuning the surface chemistry, providing a uniform passivation layer without using any complex chemistry and/or lengthy surfactants that could complicate carrier dissociation and transport, as required for application devices.

Furthermore the ns-laser processing induces the formation of Si-ncs in close proximity to each other and embedded in larger spherical particles that exceed a diameter of more than $300 \mathrm{~nm}$. We demonstrate that the close proximity of Si-ncs is essential for $\mathrm{CM}$ through space-separated quantum cutting effects (SSQC).

\section{EXPERIMENTAL DETAILS}

Quantum confined Si-ncs were produced by electrochemical etching of a silicon wafer and subsequent mechanical pulverization $[9,10]$. In order to induce $3 \mathrm{D}$ surface engineering of the surfactant-free Si-ncs and allow for the formation of Si-ncs in close proximity to each other, a ns-L processing technique has been applied. For the laser-based treatment, $2.5 \mathrm{mg}$ of the electrochemically etched Si-ncs powder was dissolved in $10 \mathrm{~mL}$ of water before being processed by the ns-L [7]. Since the Si-ncs are hydrophobic, processing in water required adding a small amount of ethanol (10 drops) to wet the surface of the Si-ncs and aggregates; this allowed producing a homogeneous colloid also in water. Laser-based surface engineering was conducted by irradiating the solution with a pulsed laser (Kr:F, $245 \mathrm{~nm}, 20 \mathrm{~Hz}, 130$ $\mathrm{mJ} /$ pulse) at room temperature for 35 minutes. In order to induce the formation of larger spherical particles containing Si-ncs, the pulse energy was increased to $145 \mathrm{~mJ} /$ pulse and the irradiation was prolonged to $55 \mathrm{~min}$. The laser beam was focused onto a $3 \mathrm{~mm}$ diameter spot on the liquid surface by a lens with a focal length of $250 \mathrm{~mm}$. During the irradiation, the glass container was closed and rotated.

The photoluminescence (PL) and the corresponding PL external quantum yield (QY) were measured using an integration sphere at room temperature. The QY is determined as the ratio of the number of emitted photons (integrated PL signal, which is the increase of signal in the region of 
photoluminescence compared with that of a reference sample) and the number of absorbed photons (integrated decrease in signal from the excitation source).

\section{RESULTS AND DISCUSSION}

Control and characterization of Si-ncs surface characteristics represent the ultimate challenge to clarify the role of all the different concurrent quantum confinement effects as the surface-to-volume ratio increases with decreasing size of the Si-ncs below $5 \mathrm{~nm}$. We showed that the rate of carrier relaxation and PL-QY can be strongly influenced by the Si-ncs surface conditions $[7,8]$. Contrary to ns-L processing where the increase in the PL-QY is rather modest (30-40\%), a drastic increase in the QY for more than 10 times is observed after DC microplasma treatment $[7,8$, $11]$.
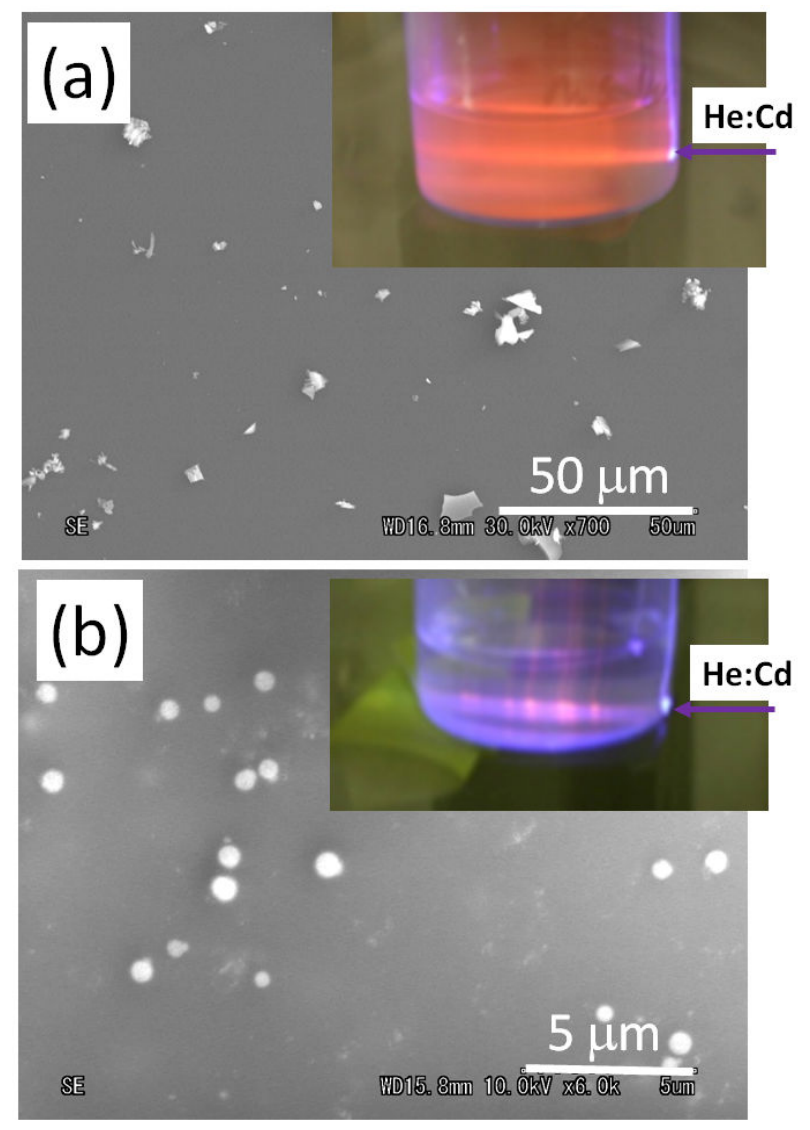

Fig. 1. Typical SEM images of electrochemically etched silicon nanocrystal: (a) as-prepared before ns laser induced surface engineering. (b) SEM images of Si-ncs spherical particles after laser irradiation of Si-ncs) powder in water. Insets in Fig 1 (a) show photograph of red light emission from Si-ncs as-prepared by electrochemical etching and inset in Fig 1 (b) corresponding colloidal solution after ns laser irradiation for $55 \mathrm{~min}$ excited in bottom (b). Arrows indicate position of excitation. In all cases the Si-ncs were excited by a He:Cd laser at $325 \mathrm{~nm}$
Pump and probe measurements confirmed that in our Si-ncs, both for as-synthesized Si-ncs and after the different surface engineering approaches, MEG is not occurring; the PL dynamics remains relatively slow in the range of 10 microseconds.. Nevertheless we have been able to observe that efficient CM does take place within closely packed Si-ncs through SSQC. Therefore the relative distance between adjacent Si-ncs play an important role for CM via SSQC. Figure 1 shows typical scanning electron microscope (SEM) images of electrochemically etched Si-ncs. As-prepared Si-ncs before ns-L surface engineering are well separated, see figure 1(a). Figure 1(b) reports SEM images of Si-ncs after ns-L processing in water that leads to the formation of large spherical particles with diameter exceeding $300 \mathrm{~nm}$ and that still contain quantum confined Si-ncs.

The specific surface chemistry together with selective pulsed heating due to laser irradiation is possibly the source of the mechanisms that are responsible for the formation of the larger spherical particles. These conditions would allow, for instance, Ostwald ripening to take place [12] whereby large particles tend to grow at the expense of smaller Si-ncs that are either synthesized by laser ablation or fragmented from electrochemically etched Si-ncs aggregates. In Ostwald ripening, it is the surface energy that determines the diffusion of small Si-ncs through the solution and allows their attachment to the surface of larger growing particles. Herein, it should be noted that Ostwald ripening is characterized by the gradual dissolution of small particles.

It has to be noted that after laser processing leading to selfassembled large spherical particles, the room temperature PL properties are preserved. The PL properties of the colloids before and after ns-L processing are shown in figure 2 and confirm that quantum confined Si-ncs are embedded in the larger spherical particles observed in figure 1(b). Inset in figure 1(a) is a photograph of the solution before processing when excited by a laser beam (He:Cd laser, indicated by an arrow). Si-ncs are well dispersed in this case and the colloid shows bright red-orange PL. Si-ncs caused substantial light scattering through the solution and a broad PL spectral envelope could be observed. However, after processing, the PL properties of the solution disappeared and no visible PL was observed when the excitation by the laser was pointed towards the middle of the solution. However PL could be measured when the laser was pointed towards the bottom of the glass ware (glass ware slightly tilted in the inset of figure 1(b)). While small size Si-ncs can easily be suspended in water to form a colloid (inset of figure 1(a)), the large spherical particles $(\sim 300 \mathrm{~nm}$ diameter) that contained the Si-ncs produced a sediment at the bottom of the solution. When the solution of the processed Si-ncs was drop-cast on a substrate brightly luminescent spots could be observed indicating the presence of the highly luminescent Si-ncs contained in the spherical larger aggregates. 
Figure 2 represents PL spectra of as-prepared Si-ncs and ns$\mathrm{L}$ processed Si-ncs which were dispersed on silicon substrates. In both cases, the measurements were performed in an integration sphere with the excitation wavelength at $360 \mathrm{~nm}$. In both cases the PL maxima is located at $620 \mathrm{~nm}$. Due to the self-assembly and the induced re-absorption, the PL intensity of the treated Si-ncs is lower. It is possible that during ns-L processing, some defects have been generated and that this may have also contributed to a lower PL intensity.

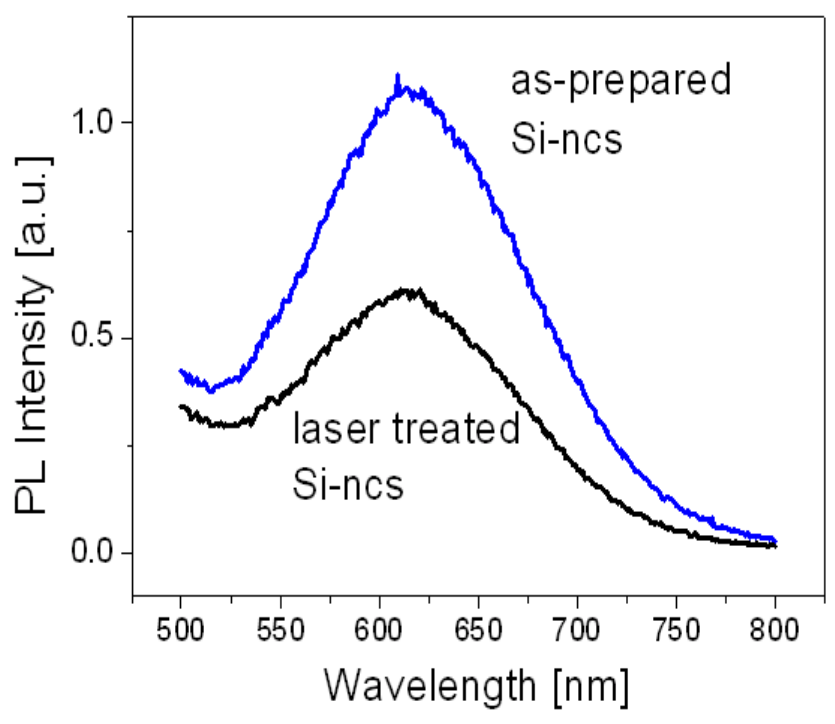

Fig. 2. Photoluminescence spectra of Si-ncs as prepared and treated by ns laser processing dispersed on silicon substrate and messured in integration sphere. In both cases the excitation wavelength was $360 \mathrm{~nm}$.

The assessment of MEG through fast fs spectroscopy is important and demonstrated MEG mechanisms in direct band gap nanocrystals [4]; however, this spectroscopy technique cannot provide a complete description of $\mathrm{CM}$ and excitons generated in a Si-nc with slow decay dynamics (i.e. microseconds) $[5,6]$. Excitons generated by high photonenergies in quantum confined Si-ncs (in our case the bandgap is about $1.8 \mathrm{eV}$ ) that do not undergo fast Auger recombination can interact with adjacent Si-ncs leading to the formation of space-separated multiple excitons through the so called SSQC [5]. In this case measurements of absolute PL quantum yield can provide additional details about $\mathrm{CM}[5,6]$. The spectral dependence of the external PL-QY of the Si-ncs was determined experimentally by comparing the total number of emitted and absorbed photons in the investigated samples [13].

Figure 3 show typical dependence of the PL-QY on excitation photon energy for the as-prepared Si-ncs colloid. The increasing trend of the QY suggests that SSQC and CM is taking place [6]. In impact ionization, the difference in effective mass of holes and electrons could be one of the most important factors determining the efficiency and threshold energy for CM. In the specific case of Si-ncs, carriers effective mass differ by a factor of two allowing for efficient CM processes. Due to the optical selection rule, photo-excited electrons and holes in Si-ncs have different excess energies that may facilitate and enhance energy transfer across neighboring Si-ncs more than within the same Si-nc. An increase of induced absorption intensity, in combination with the absence of Auger-related components in the Si-ncs dynamics, is evidence that $\mathrm{CM}$ occurs at high pumping photon energy however does not considerably affect the interaction between generated carriers. Independently, the possibility of SSQC also comes from the the slow PL decay $(\sim 24 \mu \mathrm{s})$ in our $\mathrm{Si}$-ncs which also indicates that $\mathrm{CM}$ might take place across neighboring Si-ncs.

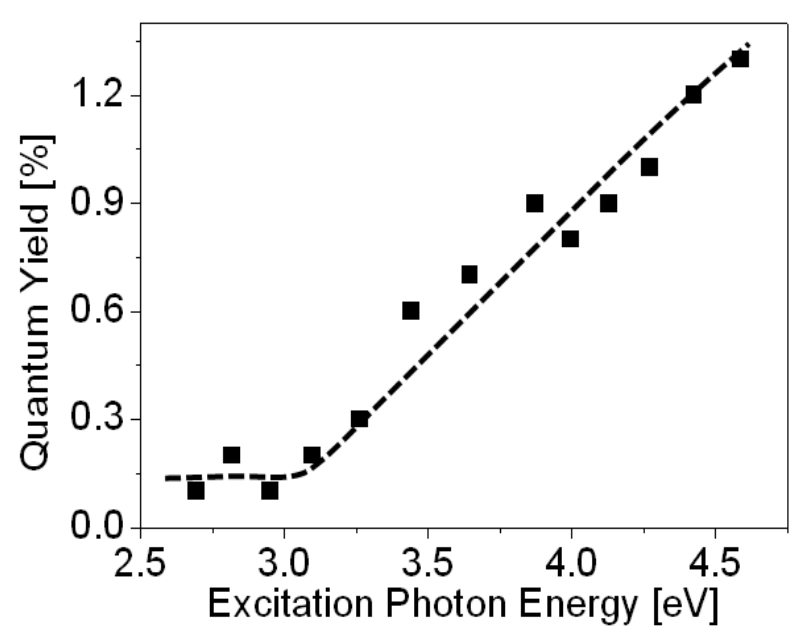

Fig. 3.The quantum yield (QY) of PL as a function of excitation photon energy for the as prepared silicon nanocrystals dispersed on silicon substrate.

In order to have a practical impact in photovoltaics, $\mathrm{CM}$ must occur with a threshold energy as close as possible to $2 E_{g}$ ( $E_{g}$ being the bandgap), and with high-efficiency (step-like) characteristics. CM can be observed [11] when the energy of an exciting photon exceeds the threshold of a multiple of the bandgap energy; in this case two or more electron-hole pair can be produced, increasing the free carrier population. Because part of the free carrier population undergoes radiative recombination, this will enhance the PL intensity and thus increase the external QY. The step-like character of the QY can therefore be used to identify this mulctiplication process (figure 4). Therefore our results suggest that the close proximity of Si-ncs within the laser-produced spherical particles could justify the step-like character of the QY and the enhancement of carriers multiplication processes. 


\section{REFERENCES}

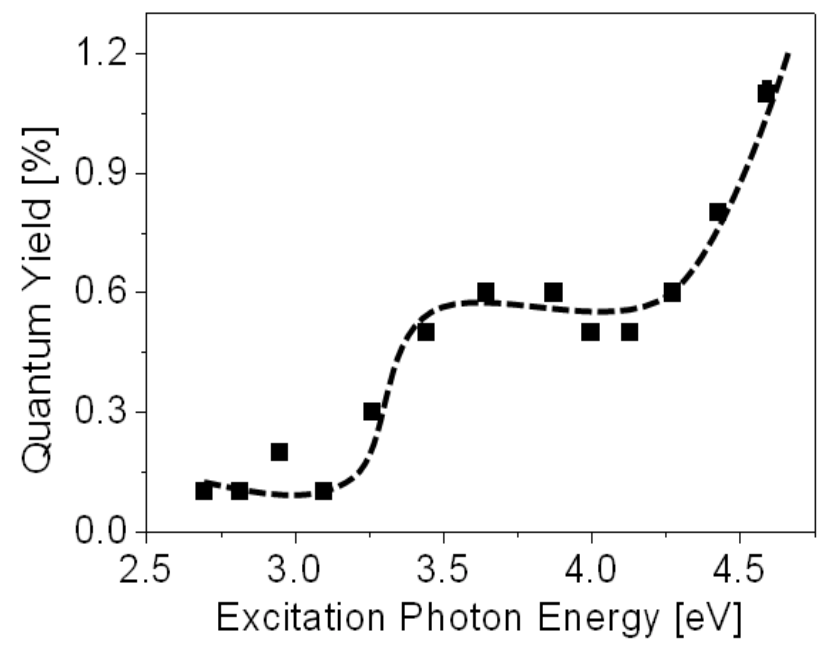

Fig. 4.The quantum yield (QY) of PL as a function of excitation photon energy for the spherical particles based on silicon nanocrystals after ns laser processing and dispersed on silicon substrate.

\section{CONCLUSION}

It is clear that the control and characterization of Si-ncs surface characteristics represent the ultimate challenge to probe and clarify the role of $\mathrm{CM}$ as the surface-to-volume ratio increases with decreasing size of the Si-ncs. The rate of carrier relaxation and PL-QY can be strongly influenced by the surface conditions. We have demonstrated that the influence of 3D surface engineering of surfactant-free Si-ncs by ns-L processing can result in ensembles of neighboring Si-ncs with efficient CM via SSQC. This synthesis approach followed by surface engineering of doped Si-ncs can, in principle, lead to innovative all-inorganic spherical solar cell architectures preserving the unique characteristics of quantum confined Sincs. Third generation all-inorganic devices will be highly desirable to harvest solar energy under high excitation intensities which, at the same time, could considerably enhance the rates of $\mathrm{CM}$ and solar cell efficiency.

\section{ACKNOWLEDGEMENT}

This work was partially supported by a NEDO Project (Japan). D.M. gratefully acknowledges the support of the JSPS Invitation Fellowship (Japan).
[1] N. M. Gabor, Z. Zhong, K. Bosnick, J. Park, \& P.L. McEuen,," Extremely Efficient Multiple Electron- Hole Pair Generation in Carbon Nanotube Photodiodes," Science, vol. 325, pp. 13671371, 2009.

[2] R. D. Schaller and V. I. Klimov "High Efficiency Carrier Multiplication in PbSe Nanocrystals: Implications for Solar Energy Conversion, " Phys. Rev. Lett. 92, pp. 186601-186605, 2004.

[3] M. C. Beard, K.P. Knutsen, P. Yu, J. M. Luther, Q. Sing, W.K. Metzger, R.J. Ellingson, A.J. Nozik, "Multiple Exciton Generation in Colloidal Silicon Nanocrystals _" Nano Lett. Vol. 7, pp. 2506-2512, 2007.

[4] A. Nozik et al., "Multiple exciton generation in semiconductor quantum dots", Chem. Phys. Lett. Vol.457, pp. 3-10, 2008.

[5] D. Timmerman I. Izeddin, P. Stallinga, I. Yassievich, T. Gregorkiewicz, "Space-separated quantum cutting with silicon nanocrystals for photovoltaic applications", Nat. Photonics, vol. 2, pp. 105-109, 2008.

[6] D. Timmerman J. Valenta, K. Dohnalová, W. D. A. M. de Boer, T. Gregorkiewicz,'Step-like enhancement of luminescence quantum yield of silicon nanocrystals, "Nature Nanotechnology;" vol. 6, pp.710-713, 2011.

[7] V. Švrcek, D. Mariotti, Y. Shibata, M. Kondo "Hybrid heterojunction based on fullerenes and surfactant-free, selfassembled, closely-packed, silicon nanocrystals", J.of Phys. D: Appl. Phys. vol. 43, pp. 415402-415409, 2010.

[8] V. Švrcek, D. Mariotti, M. Kondo, "Microplasma-induced surface engineering of silicon nanocrystals in colloidal dispersion Appl.Phys. Lett. Vol. 97, pp. 161502-1-161502-3, 2010.

[9] V. Švrcek, A. Slaoui and J.-C. Muller, "Ex-situ prepared Si nanocrystals: Their elaboration and characterization in embedded silica glass", J. Appl. Phys. vol. 95, pp. 3158-3164, 2004.

[10] V. Švrcek, H. Fujiwara, M. Kondo,"Top-down silicon nanocrystals and a conjugated polymer-based bulk heterojunction : Optoelectronic and photovoltaic applications", Acta Materialia, vol. 57, pp. 5986-5994, 2009.

[11] D. Mariotti, V. Švrcek, W. J. Hamilton. M. Schmidt, M. Kondo, "Silicon nanocrystals in liquid media: Optical properties and surface stabilization by microplasma induced non equilibrium liquid chemistry”, Adv. Funct. Mat. Vol. 22 pp. 954-962, 2012.

[12] L. Ratke \& P.W. Voorhees, "Growth and Coarsening: Ostwald Ripening in Material Processing," New York, Springer Press, 2002.

[13] J. C. de Mello, H. F. Wittmann, \& R. H. Friend, An improved experimental determination of external photoluminescence quantum efficiency. Adv. Mater. Vol. 9, pp.230-232, 1997. 